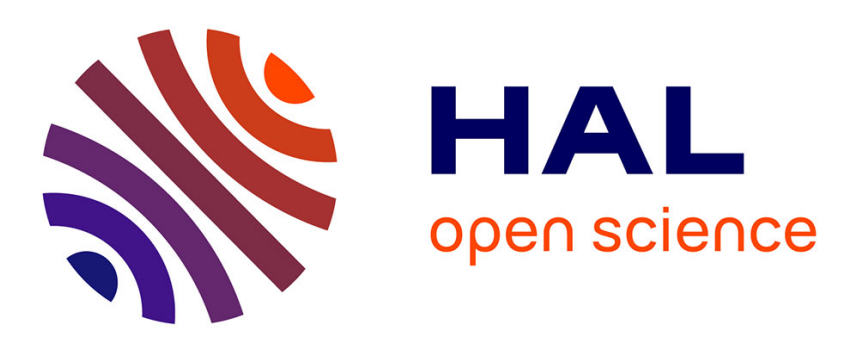

\title{
Contribution of somesthetic information to the perception of body orientation in the pitch dimension.
}

Lionel Bringoux, Vincent Nougier, Pierre-Alain Barraud, Ludovic Marin, Christian Raphel

\section{- To cite this version:}

Lionel Bringoux, Vincent Nougier, Pierre-Alain Barraud, Ludovic Marin, Christian Raphel. Contribution of somesthetic information to the perception of body orientation in the pitch dimension.. The Quarterly Journal of Experimental Psychology: Section A, 2003, 56 (5), pp.909-23. 10.1080/02724980245000016 . hal-00193896

\section{HAL Id: hal-00193896 https://hal.science/hal-00193896}

Submitted on 10 Feb 2020

HAL is a multi-disciplinary open access archive for the deposit and dissemination of scientific research documents, whether they are published or not. The documents may come from teaching and research institutions in France or abroad, or from public or private research centers.
L'archive ouverte pluridisciplinaire HAL, est destinée au dépôt et à la diffusion de documents scientifiques de niveau recherche, publiés ou non, émanant des établissements d'enseignement et de recherche français ou étrangers, des laboratoires publics ou privés. 


\title{
Contribution of somesthetic information to the perception of body orientation in the pitch dimension
}

\author{
Lionel Bringoux and Vincent Nougier \\ Université Joseph Fourier, Grenoble, France \\ Pierre-Alain Barraud \\ Centre de Recherches du Service de Santé des Armées, La Tronche, France \\ Ludovic Marin \\ Université Joseph Fourier, Grenoble, France \\ Christian Raphel \\ Centre de Recherches du Service de Santé des Armées, La Tronche, France
}

\begin{abstract}
This study investigated the contribution of otolithic and somesthetic inputs in the perception of body orientation when pitching at very slow velocities. In Experiment 1, the subjects' task was to indicate their subjective postural vertical, in two different conditions of body restriction, starting from different angles of body tilt. In the "strapped" condition, subjects were attached onto a platform by means of large straps. In the "body cast" condition, subjects were completely immobilized in a depressurized system, which attenuates gravity-based somesthetic cues. Results showed that the condition of body restriction and the initial tilt largely influenced the subjective postural vertical. In Experiment 2, subjects were displaced from a vertical position and had to detect the direction of body tilts. Results showed that the threshold for the perception of body tilt was higher when subjects were immobilized in the body cast and when they were tilted backward. Experi ment 3 replicated the same protocol from a supine starting position. Compared to results of Experiment 2, the threshold for the perception of body tilt decreased significantly. Overall, these data suggested that gravity-based somesthetic cues are more informative than otolithic cues for the perception of a quasi-static body orientation.
\end{abstract}

Requests for reprints should be sent to Vincent Nougier, Université Joseph Fourier, UFRAPS, BP 53, 38041 Grenoble cedex 9, France. Email: vincent.nougier@ujf-grenoble.fr

We are grateful to the Instrumentation and Technological Research Service of the Centre de Recherches du Service de Santé des Armées for the construction of the rotating platform and the body cast, the development of electronic control, and the technical expertise and assistance. We also would like to thank the three anonymous reviewers for their helpful comments and suggestions on an earlier version of this manuscript. 
It is well known that sensory information from the visual, somesthetic, and vestibular systems contributes to the perceived orientation of the body in space. According to the ecological theory of orientation, severalauthors consider that the intermodalelationship between patterns of energy available to each of these sensory systems constitutes in itself high-order information that cannot be divided (Fouque, Bardy, Stoffregen, \& Bootsma, 1999; Gibson, 1966; Stoffregen \& Bardy,2001; Stoffregen \& Riccio,1988). On the other hand, many researchers relying on sensory conflict experiments regard the perception of body orientation as based on the integration or sensory signal fusion (Howard, 1997; Mergner \& Glasauer, 1999) of gravity-based visual, vestibular, and somesthetic information. However, within this theoretical background, the respective contribution of these sensory systems-that is, the weight attributed by the central nervous system during the integration process-is still a matter of debate. As mentioned by Mittelstaedt (1999), the perception of body orientation in total darkness is traditionally thought to be based on vestibular information and supplemented by somesthetic information. It has been proposed that otolith organs are natural graviceptive organs (Young, 1984) and provide accurate information about the direction of gravity forces and the orientation of the body with respect to them. As it was clearly expressed by Mittelstaedt (1999), in the absence of all other spatial information, and under purely static conditions, the otolith system is known permanently to produce a set of afferents from which a "virtually veridical representation of the vertical" with respect to the head may be derived. Electrophysiological evidence about modifications of the discharge of primary otolith neurons during slight head tilts (Fernandez \& Goldberg, 1976) contributed to convey the assumption that the otoliths signal not only the static orientation of the head to gravity, but also changes in orientation that accompany angular head displacements (Benson, 1990).

On the other hand, experiments conducted in microgravity emphasized the importance of some somesthetic cues for the perception of body orientation (Lackner \& DiZio, 1993, 2000). When subjects who where free floating without any contact with the aircraft closed their eyes, many of them lost all sense of body orientation in relation to their surroundings. For these subjects, applying pressure to the tops of their heads or under the soles of their feet made them feel upside down or rightside up, respectively. This clearly indicates that specifically oriented somesthetic stimulation is sufficient to convey information about body orientation in space. However, this does not give further knowledge about the predominance of these types of sensory cue over gravity-based otolithic information. These results simply showed that some somesthetic cues could adequately compensate for the lack of gravity-based otolithic informa tion, the latter still considered as the most accurate graviceptive organ on Earth.

Nevertheless, experiments carried out in water immersion have led to interesting results contrasting with the previous conclusion (Browr,961; Massion,Fabre, Mouchnino, \& Obadia, 1995; Nelson, 1968; Ross, Crickmar, Sills, \& Owen, 1969). During immersion and in a buoyant statethe somesthetic system has a limited access to gravity-based information, whereas gravity still acts on a component of the vestibular system (Ross, 1990). Therefore, in such an environmental condition, the specific contribution of otolithic information to the perception of body orientation in space can be more directly studied.Subjects'postural responses to the vertical under water were strikingly inaccurate, showing a forward tilt ranging from 7.3 deg (Massion et al., 1995) to 13.2 deg (Ross et al., 1969). This suggests that gravitybased somesthetic cues may play a more important role than the otoliths for perceiving a static body orientation. 
However, methodological criticisms were often associated with these experiments (e.g., Stoffregen \& Riccio,1988). For instance, fixation straps or shoes (Massion et al1,995;

Nelson, 1968) may generate tactile cues that are sometimes perturbing with respect to the task requirements. When subjects were not fixed onto a platform, judgements were performed after rapid whole-body rotations (Brown, 1961; Ross et al., 1969), which could lead to strong semicircular aftereffectsoverall, severalaspects can disturb subjects' perception under water, such as fluid resistance to body displacement, the relative buoyancy of the body, or the respiratory equipment (Brown, 1961). Therefore, other experimental methods must be used to avoid the undesirable effectsof the studies conducted in immersion aswell as in microgravity.

The present experiments were built in the frame of the previous conclusion for further investigating the respective contribution of the vestibular and somesthetic systems to quasistatic spatiabrientation.More specifically,we analysed the extent to which subjects can consciously perceive their body orientation, when gravity-based tactile and proprioceptive cues are either available or attenuated by means of a specific body cast.

In Experiment 1, the subjects' task was to indicate their subjective postural vertical, starting from different angles of body tilts in a forward or backward initial position. In Experi ment 2, the subjects' task was to detect a very slow body tilt in the pitch dimension with respect to a referred vertical body orientation. In Experiment 3, instead of starting from a vertical position, subjects were initially oriented in a supine position and had to detect a tilt from this initial orientation. For each experiment, the task was executed in two conditions of body restriction. With regard to somesthetic implications enlightened in microgravity and underwater experiments, our main hypothesis postulated that errors of estimation in body orientation increased when body restriction increasedbecause oftactile and proprioceptive alteration.Moreover, it was expected that the direction of tiltas wellas the initial body orientation, might lead to differences in spatial orientation judgements, mainly because of interoceptive influences (Mittelstaedt, 1992, 1999).

\section{GENERAL METHOD}

In all experiments reported here, subjects were restrained on a servo-controlled motorized platform (Figure 1) allowing backward and forward rotations in the sagittal plane (pitch dimension) at a constant velocity of 0.05 deg s, following an initial acceleration phase at 0.005 $\operatorname{deg} \mathrm{s}^{2}$. Moving the platform at this very slow velocity prevented responses of the semicircular canals (Goldberg \& Fernandez, 1984). Position signals from the platform were sampled at 20 $\mathrm{Hz}$ (12-bit A/D converter). Earphones providing white noise masked the noise induced by the motor during rotation.

Two conditions of body restriction were manipulated (strapped and body cast). In the socalled strapped condition (Figure 1), subjects were attached onto the platform by means of six large straps fixed at the level of the head, shoulders, chest, hips, knees, and ankles. In the body cast condition, subjects were completely immobilized in a specific device looking like a body cast. This body cast was composed of three plastic envelopes full of polystyrene micromarbles and connected to a depressurization device. The surface of the plastic envelopes was very thin in order to ensure the closest contact possible with the subjects' body. The wrapping was done in the supine posture with no contact of the soles with the foot-rest. The back envelope was 


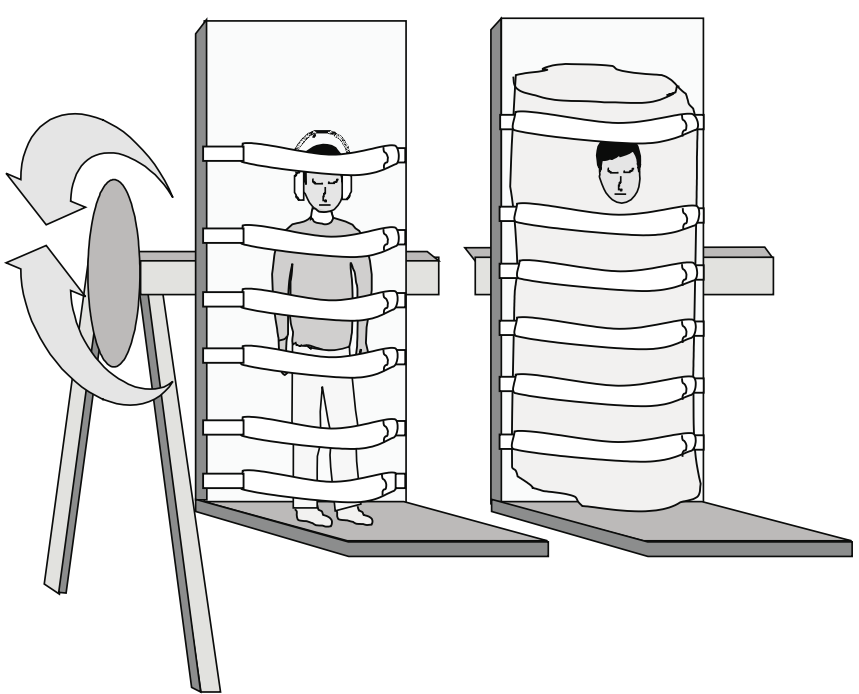

Figure 1. Illustration of the experimental set-up. The motorized platform rotated around the central axis. In the strapped condition (left), subjects were attached onto the platform by mean of six large straps fixed at the level of the head, shoulders, chest, hips, knees, and ankles. In the body cast condition (right), subjects were completely immobilized in a depressurized body cast fixed onto the platform.

directly attached onto the platform and entirely covered the back of the subjects' body from the head to the feet. The front envelope was put onto the front of the subjects' body from the neck to the feet and was maintained by means of the straps. The third envelope specifically immobilized the head and shoulders. When subjects were appropriately wrapped, the body cast was depressurized.

A preliminary experiment was conducted to investigate the capability of the body cast to minimize the gravity-based tactile stimulation-that is, to create a more diffuse and isotropic pattern of pressure around the body and to suppress important changes in pressure repartition that occur for different body tilts.

Two subjects (one male, $1.74 \mathrm{~m}, 76 \mathrm{~kg}$; one female, $1.62 \mathrm{~m}, 58 \mathrm{~kg}$ ) were restrained on the movable platform in the two strapped and body cast conditions. Subjects were tilted at pitch angles ranging from -90 deg to +90 deg by steps of $15 \mathrm{deg}$. Pressure variations were recorded at six different parts of the body by means of force sensing resistors. The variation of resistance as a function of force gives an estimation of the variation of pressure generated at the level of the captor, with the output resistance increasing as the force decreases. The six resistor loca tions were distributed as follows: three on the posterior part of the body (D-10 vertebra, ischiatic tuberosity, and left gastrocnemius proximal insertion), two on the anterior part of the body (substernal central point and patella base), and one under the feet (first phalanx of the left big toe).

As illustrated in Figure 2, results obtained from the two subjects did not differ signifi cantly. This observation showed thathe different morphologicalcharacteristics of he subjects do not interact with the distribution and evolution of pressure cues with respect to body orientation. 

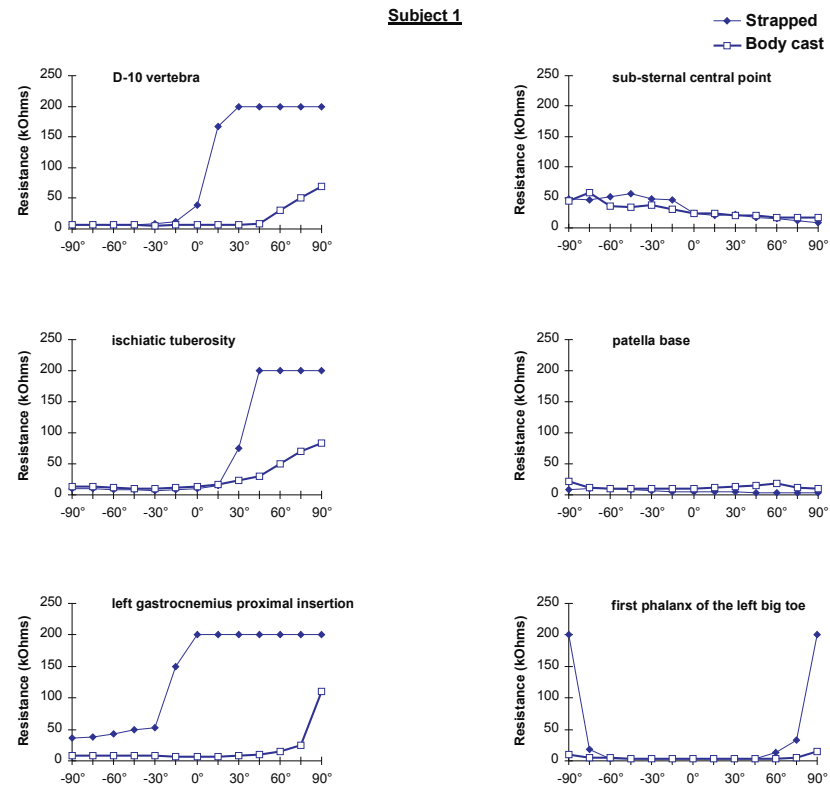

$\underline{\text { Subject } 2}$
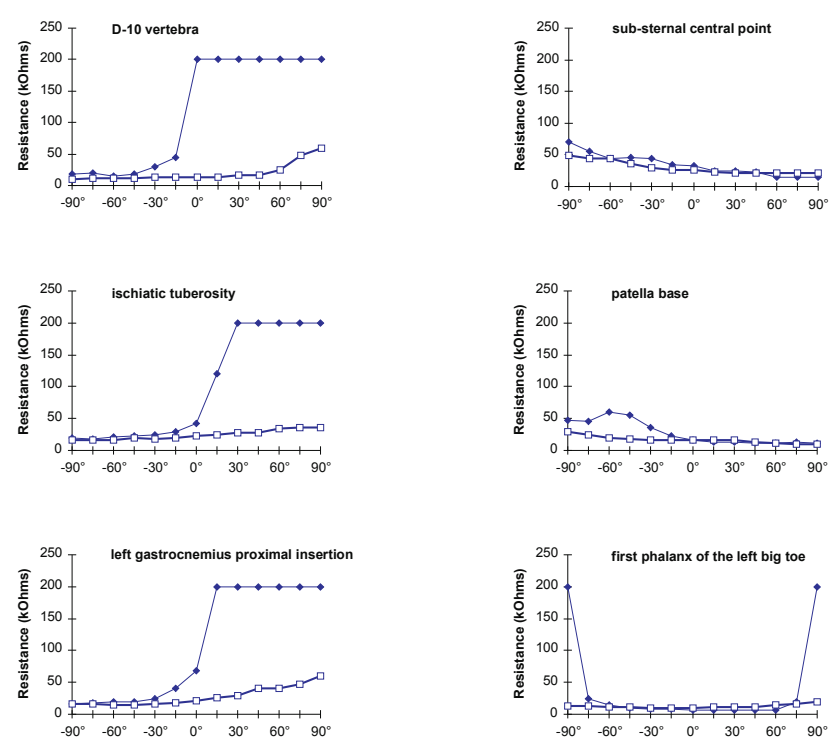

Figure 2. Variation of the resistance of the sensors as a function of body tilt at six different body locations for the two support conditions (strapped and body cast) and the two subjects (a and b); -90 deg, corresponded to an horizontal supine body orientation, 0 deg to a vertical body orientation, and +90 deg to a horizontal prone body orientation. The D-10 vertebra, ischiatic tuberosity, and left gastrocnemius proximal insertion corresponded to the location of the force-sensing resistors on the posterior part of the body, the substernal central point and patella base to the location of the resistors on the anterior part of the body, and the first phalanx of the left big toe to the resistor located under the feet. 


\section{BRINGOUX ET AL.}

Regarding the variations of resistance measured by the force-sensing resistors at the six body locations, there was no difference between the two conditions of body restriction for two locations only:the substernacentral point and the patella base. This fact can be easily explained considering that the force-sensing resistors placed at the anterior part of the body in the strapped condition were fixed just under the tightened straps. To some extent, it recreated locally the body cast condition.

Under the sole of the feet (first phalanx of the left big toe), resistance values differed for extreme body tilts only ( $> \pm 60 \mathrm{deg})$. When the subjects were tilted over 60 deg towards the horizontal supine or prone body orientation, a take off occurred in the strapped con dition, leading to no contact,while pressure remained nearly constant in the body cast condition.

Regarding the posterior part of the body, the resistance level was almost identical for both conditions of body restriction for the backward angles of tilt. However, in the strapped condition, the resistance values increased abruptly near the vertical to reach infinity for the forward tilt angles.Conversely,in the body cast condition, the resistance values still remained nearly constant or slowly increased when the body was tilted forward, over the vertical. These data clearly showed that the posterior parts of the body took off from the platform in the strapped condition, unlike in the body cast condition. This event occurred at different angles of tilt around the vertical, according to each body location. It began by the left gastrocnemius proximal insertion at approximately -15 deg backwards followed by the $\mathrm{D}-10$ vertebra just around the vertical, and then by the ischiatic tuberosity around $+30 \mathrm{deg}$ forwards.

To summarize, in the strapped condition, slight tilts led to a consistent modification in the repartition of pressure exerted on the body by the support surface. Gravity-based tactile and proprioceptive cues were then still clearly present. In the body cast condition, however, pressure generated by antigravity reaction forces was still present but drowned in a wholeisotropic pattern of pressure stimulation, which was nearly invariant whatever the body tilt. Hence, if the body cast condition could not obviously be assimilated to a tactile deprivation situation, the gravity-based tactile cues were nevertheless less discernable for the central nervous system. Moreover, because of the suppression of postural activity, whatever the body orientation, gravity cues sensed by "load" receptors were no more available (Dietz, Gollhofer, Kleiber, \& Trippel, 1992). Consequently, compared to the strapped condition, it can be stated that gravity-based somesthetic cues were attenuated in the body cast.

\section{EXPERIMENT 1}

The purpose of Experiment 1 was to investigate the respective vestibular and somesthetic contribution to the judgement of the subjective posturalvertical. In this classical task (Bisdorff, Wolsley,Anastasopoulos, Bronstein \& Gresty, 1996;Clark \& Graybiel,1963), subjects were asked to estimate their perceived verticality, starting from various tilted posi tions in the pitch dimension. 


\section{PERCEPTION OF BODY ORIENTATION}

\section{Methods}

\section{Subjects}

A total of 10 subjects ( 7 males and 3 females, mean age $=27$ years) participated in Experiment 1 . All subjects gave signed informed consent in compliance with the ethical committee (i.e., Helsinki Conven tion), which governs and regulates human experimentation in France. As in the following experiments, none of these subjects reported any sensation of discomfort, which could interfere with their judgement skills.

\section{Procedure}

The subjects' task was to verbally indicate when they felt vertical in the pitch dimension in total dark ness. The two conditions of body restriction presented above were investigated in two counterbalanced sessions. Each session was composed of six trials crossing randomly two directions of initial orientation (forward and backward) $\times$ three angles of initial orientation (10, 20, and $30 \mathrm{deg})$. Subjects were first tilted to the desired initial orientation at a constant velocity of $4 \mathrm{deg}$ s. After $20 \mathrm{~s}$, allowing the information issued from the semicircular canals to be close to zero (Goldberg \& Fernandez, 1984), the tilting platform started its slow displacement $(0.05 \mathrm{deg} \mathrm{s})$ back to the direction of the gravitational vertical. Subjects were encouraged to give their response with a confidence level of minimum 4 on a 5-point scale. At the end of each trial-that is, after the subjective postural vertical was judged, subjects were placed in the upright position at a random velocity, to avoid any estimation of the performance, and the lights turned on for $30 \mathrm{~s}$, to avoid any postural after effect or disorientation illusion prior to the next trial. No verbal feedback about performance was given at any time. Furthermore, subjects were not instructed either about the direction and magnitude of the initial orientation, or about the angular velocities of platform rotation. For making their judgement, subjects were strongly encouraged to use available body sensations rather than making inferences about the time they spent during one trial.

\section{Results and discussion}

The mean absolute error of judgement with respect to gravity was used to express the subjec tive postural vertical, independently of the signed direction of the body tilt. A 2 (conditions of body restriction: strapped and body cast) $\times 2$ (directions of initial tilt: forward and backward) $\times 3$ (angles of initial tilt: 10,20, and $30 \mathrm{deg}$ ) analysis of variance (ANOVA) with repeated measures on the three factors was applied to the data. Results showed that the mean error of judgement was smaller in the strapped than in the body cast condition (4.19 deg \pm 3.10 vs. 6.49 deg \pm 4.86 , respectively), $F(1,9)=9.94, p<.05$, accounting for $20.3 \%$ of the variance. There was also a main effect of the angle of initial tilt, $F(2,18)=25.87, p<.001$, accounting for $57.4 \%$ of the variance, but no effect of the direction of this initial tilt. A post hoc analysis (Scheffé test) showed that the error of judgement increased from 10 to $30 \mathrm{deg}(3.1 \mathrm{deg} \pm 1.0,5.1 \mathrm{deg} \pm 2.3$, and 7.9 deg \pm 3.0 , for the 10 -, 20 - and 30 -deg tilted positions, respectively, $p s<.05$ ). Observa tion of the data showed that the errors of judgement consistently deviated in the direction of the initial tilt. This effect increased when increasing the angle of tilt. Finally, there was also a significant interaction of body restriction by angle of initial tilt, $F(2,18)=6.66, p<.01$. As illustrated in Figure 3 , the effect of the angle of initial tilt was greater in the body cast than in the strapped condition.

It is well known that both normal and labyrinthine defective subjects are able to give fairly accurate estimates of the subjective postural vertical, with errors smaller than 2 
BRINGOUX ET AL.

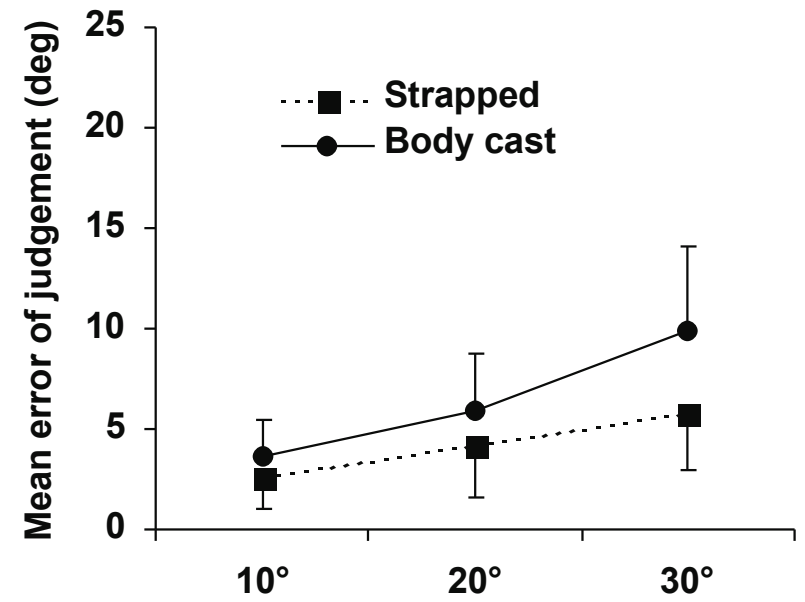

Figure 3. Mean error of subjective postural vertical judgement with respect to gravity and standard deviation for the two conditions of body restriction and the three angles of initial body tilt (Experiment 1 ).

degrees, when gravity-based somesthetic cues are still available (Anastasopoulos, Haslwanter, Bronstein, Fetter, \& Dichans, 1997; Bisdorff et al., 1996; Clark \& Graybiel, 1963). The present results clearly suggested that the somesthetic system plays a major role in estimating the direction of subjective postural vertical (Bronstein, 1999). The greater the limitation of the contribution of the somesthetic system, the greater was the error of judgement with respect to the gravitational vertical and to the initial angle of tilt. This also indicates that the otoliths are unable to compensate for the lack of gravity-based tactile and proprioceptive information.

In a task involving subjective postural vertical judgements, subjects had to associate a specific multisensory configuration to a particular postural orientation. Previous authors proposed that the central nervous system incorporates information about body dynamics and sensory dynamics to develop an internal model (Lestienne \& Gurfinkel, 1988; Merfeld, Young, Oman, \& Shelhamer, 1993; Merfeld, Zupan, \& Peterka, 1999). The output of this internal model-thatis, expected sensory afferences for a particular body position-is compared to the actualavailable sensory afferences. The error between this perceptual internal reference developed through subjects' experience (BringouxMarin, Nougier, Barraud, \& Raphel, 2000) and the actual sensory afferences would be used to drive the central estimate towards the value of reference. In the absence of enough somesthetic information about body orientation with respectto their own reference of verticality (Lestienne \& Gurfinkel, 1988), the subjects tended to perceive their current body position as nearer to the upright reference position than it was in reality.

The same argument can explain the differences observed with respect to the angle of initial tilt. Because of the very slow rotation of the platform, changes in the somesthetic cues over time are very low leading to an underestimation of the current body tilt (Higashiyama \& Koga, 1998). As previously indicated, this underestimation increased in the body cast condition (Figure 3). 


\section{EXPERIMENT 2}

The purpose of Experiment 2 was to investigate the respective contribution of gravity-based somesthetic and vestibular cues in the perception of a slow body tilt from an initial vertical body orientation. This task can be considered to some extent as the reverse of the previous subjective postural vertical task, subjects having to perceive a tilt from a vertical position instead of having to perceive the vertical from a tilted position. Threshold for the perception of a change in body orientation was defined for both conditions of body restriction and both directions of body tilt.

\section{Method \\ Subjects}

A total of 8 new subjects ( 5 males and 3 females, mean age $=26$ years) participated in the study. All subjects gave informed consent and were naive about the purpose of the experiment.

\section{Task and procedure}

The subjects' task was to perceive backward and forward slow body tilts in total darkness, starting from an initial vertical body orientation. Prior to each trial, subjects were oriented in the initial position. When ready, the trial was initiated, and it ended with the detection of a body tilt. It has been emphasized elsewhere (Fitzpatrick \& McCloskey, 1994; Teasdale et al., 1999) that the detection of a body tilt can occur before the direction of the pitch can be reported. Subjects were instructed to indicate verbally when they perceived a change in body orientation and the direction of tilt. In order to obtain responses that exceeded that of simple chance, subjects were also instructed to give their level of confidence on a 5point scale. They were encouraged to postpone their response until reaching Level 4 on the 5-point scale. Following the response, the platform was brought back to the starting position at a random velocity to avoid any estimation of the performance. Furthermore, no verbal feedback about subjects' performance was given at any time. The two conditions of body restriction were tested in two separate blocks of trials presented randomly. For each condition, 4 trials for each pitch direction (backward and forward) and 2 catch trials with the platform remaining immobile were executed randomly for a total of 10 trials. The catch trials were used to further ensure that subjects did not detect movements of the platform by guessing. The duration of these trials varied randomly from 120 to $300 \mathrm{~s}$, prior to indicating to the subjects that a new trial would be initiated. When an error of judgement was made, an additional identical trial was randomly inserted in the block. Therefore, subjects were not told either about the number of trials that composed a block, or about the direction of tilt.

\section{Results and discussion}

Errors of judgemenĐverall, subjects made judgement errors in $10.5 \%$ and $24.5 \%$ of the trials in the strapped and body cast conditions, respectively. Among these errors, $30 \%$ were due to a wrong perception of the direction of tilt during an effective platform rotation; the $70 \%$ remaining errors were due to a wrong perception of body tilt during catch trials. In all cases, the erroneous reported direction of tilt was different across trials and subjects.

Detection thresholds. A 2 (body restriction: strapped, body cast) $\times(2$ pitch direction: back ward, forward) ANOVA with repeated measures on all factors was applied to the threshold 
for the perception of a body tilt. Results showed main effects of body restriction, $F(1,7)=$ $80.12, p<.001$, accounting for $55.1 \%$ of the variance, and direction, $F(1,7)=51.95, p<.001$, accounting for $37.5 \%$ of the variance. Threshold for the perception of a body tilt was lower in the strapped than in the body cast condition ( $7.6 \mathrm{deg} \pm 1.6 \mathrm{vs}$. $15.8 \mathrm{deg} \pm 1.6$, respectively). It was also lower when the platform pitched forward than when it pitched backward ( $8.3 \mathrm{deg} \pm$ 1.5 vs. $15.1 \mathrm{deg} \pm 1.7$, respectively). Results also showed a significant two-way interaction of body restriction by direction, $F(1,7)=13.94, p<.01$. As illustrated in Figure 4, the effect of restriction was greater when pitching backward than when pitched forward.

Results of the present experiment showed that the threshold for the perception of a body tilt was higher when subjects were completely immobilized in the body cast than when partially restrained. Once again, gravity-based tactile and proprioceptive sensory cues seemed to play a major role in the perception of body orientation. The very high threshold observed in the body cast condition supported the results of various authors (Fouque et al., 1999; Riccio, Martin, \& Stoffregen, 1992; Teasdale et al., 1999). These authors suggested that the percep tion of body orientation is disturbed when proprioceptive information is not dynamically inte grated, and that otolith organs cannot naturally convey absolute graviceptive information at a perceptual level in quasi-static conditions.

Results also showed that the threshold for the perception of a body tilt was dependent on the direction of tilt. It may be hypothesized that another part of somesthetic information is differentially involved from one direction to another. Recent experimental works have shown that information provided by internal organs could play a role in postural orientation (von Gierke \& Parker, 1994; Mittelstaedt, 1992, 1999; Mittelstaedt \& Fricke, 1988). Interoceptive cues could probably account for such a directional asymmetry already found by Nemire and Cohen (1993). The following experiment was carried out to further explore the potential role of interoception in the perception of a change in body orientation.

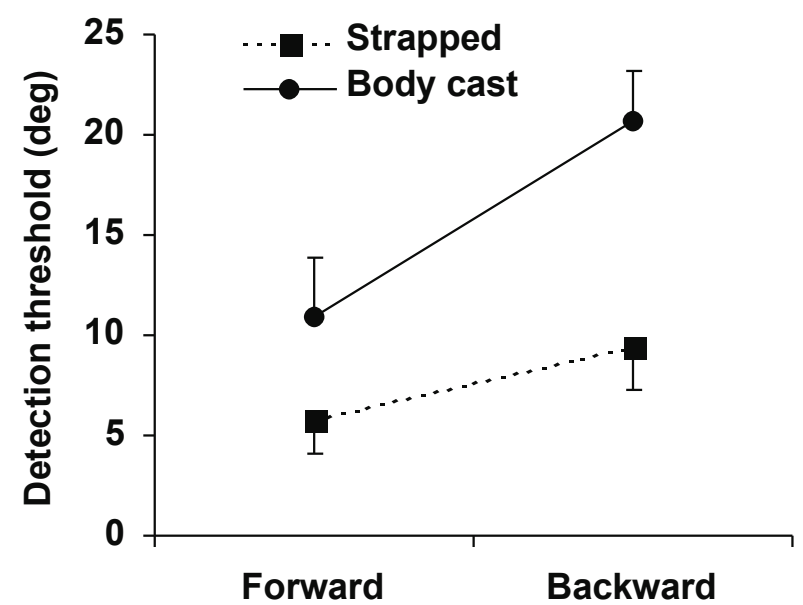

Figure 4. Mean angular threshold and standard deviation for the perception of a body tilt starting from a vertical position for the two conditions of body restriction and the two directions of tilt (Experiment 2). 


\section{EXPERIMENT 3}

To study the potential interoceptive components of somesthetic information in the perception of body tilt, we replicated Experiment 2, with subjects oriented in a supine starting position. It was expected that perception of body orientation also may be dependent on the hydrostatics of the blood (Mittelstaedt, 1992) - that is, subjects made fewer errors when the pitch rotation induced a body orientation with the head toward the bottom than when the feet were toward the bottom.

\section{Method}

\section{Subjects}

A total of 8 subjects ( 4 females and 4 males, mean age 26.2 years) participated in Experiment 3. Of these, 6 were new recruited subjects whereas 2 also took part in Experiment 2. They all gave informed consent and were naive as to the purpose of the experiment.

\section{Task and procedure}

The procedure was the same as that in Experiment 2, except that the subjects had to detect a slow change in body orientation from an initial supine position.

\section{Results and discussion}

Errors of judgement. Overall, subjects made judgement errors in $4.5 \%$ and $10.5 \%$ of the trials in the strapped and body cast conditions, respectively. Among these errors, $30 \%$ were due to a wrong perception of the direction of tilt during an effective platform rotation; the $70 \%$ remaining errors were due to a wrong perception of body tilt during catch trials. In all cases, as in the previous experiment, the erroneous reported direction of tilt was different across trials and subjects.

Detection thresholds. A 2 (body restriction: strapped, body cast) $\times 2$ (pitch direction: back ward, forward) ANOVA with repeated measures on all factors was applied to the threshold for the perception of a body tilt. Results showed main effects of body restriction, $F(1,7)=$ $10.87, p<.05$, accounting for $56.1 \%$ of the variance, and direction, $F(1,7)=6.13, p<.05$, accounting for $35.4 \%$ of the variance. Figure 5 illustrates the results obtained in Experiment 3 .

Although less consistent than in Experiment 2, the effect of body restriction led to a threshold for the perception of a body tilt that was lower in the strapped than in the body cast condition (5.2 deg \pm 1.6 vs. 7.8 deg \pm 2.1 , respectively). However, contrary to Experiment 2 , the threshold was higher when the platform pitched forward than when it pitched backward (7.6 deg \pm 2.6 vs. $5.5 \mathrm{deg} \pm 1.0$, respectively). Furthermore, observation of the data showed that the thresholds observed in Experiment 3 were lower than those in Experiment 2. These results strongly suggested that a particular source of information is better used starting from a supine position. As proposed by Mittelstaedt (1992), influence of gravity on the cardiovas cular system should be a good candidate. Variations of homeostatic pressure with body tilt around the horizontal position appear as very informative cues, which can compensate for the tactile and proprioceptive disturbances generated by the body cast. Moreover, when subjects 


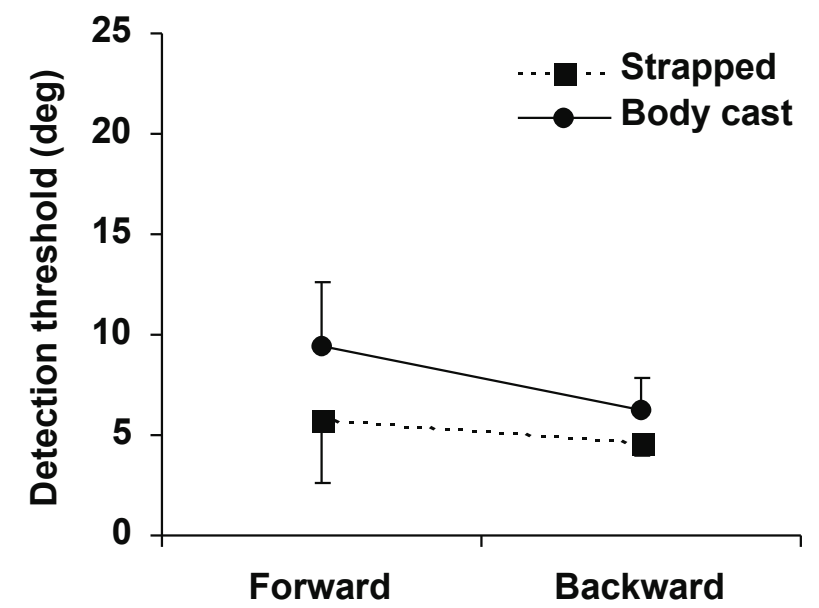

Figure 5. Mean angular threshold and standard deviation for the perception of a body tilt starting from a supine position for the two conditions of body restriction and the two directions of tilt (Experiment 3).

were tilted backward, such an unusual and alarming situation probably lowered the threshold for the perception of a body tilt, mainly because of the rush of blood to the head.

\section{GENERAL DISCUSSION}

Results of the present experiments showed that the perception of body orientation was more accurate when subjects were partially restrained than when they were completely immobilized in the body cast. In that condition, the appropriate use of gravity-based tactile and proprioceptive information was altered. The overall pattern of somesthetic stimulation gener ated by antigravity reaction forces thus appears to be highly informative for the perception of body orientation (Lackner \& DiZio, 1993, 2000).

As mentioned by Higashiyama and Koga (1998), tactile mechanoreceptors, distributed over the wide area of cutaneous tissue, appear to provide the central nervous system with spatially and temporally coded information associated with changes in the distribution of pressure on the skin that result from changes in body orientation. Thus, the imporfant contri bution of tactile mechanoreceptors to perceived body orientation suggested by our results might greatly depend on the availability of changing pressure patterns generated during body rotation (Bringoux, Schmerber,Nougier, Dumas, Barraud, \& Raphel, 2002; Lackner \& Graybiel, 1978). Similarly, changes in muscular tension opposed to the attraction of gravity, which are eliminated in the body cast, may play a major role in perceiving a body tilt. In addi tion, "load receptors" such as Golgi tendon organs have been shown to yield gravity-based information during active postural stance (Dietz et al., 1992). Thus, antigravity muscular activity might also generate cues at a perceptual level, informing the subjects about their orien tation in space. The present results also showed that when the use of visual, vestibular, tactile, and proprioceptive information is altered, sensory inputs from truncal graviceptors (Mittelstaedt, 1992,1997;Mittelstaedt \& Fricke, 1988) could be of great importance to 
perceive body orientation in space. Mittelstaedt and collaborators, on the basis of "subjective horizontal position" experiments conducted with paraplegics and nephrectomized patients on a tiltable board and on a sled centrifuge, enlightened at least two separate truncal graviceptive inputs. A first input could originate in renal afferences about pressure exerted towards the surface of the kidney. A second input has been shown to yield gravity information through the inertia of a mass in the body. The mass of blood in the large vessels, the mass of the abdominal viscera (von Gierke \& Parker, 1994), and hydrostatic effects have been respectively evoked as potential sources for this second input. Lower tilt detection threshold in supine than in vertical initial orientation, as well as the reversed effect of the direction of tilt, might be due to an increased relevance of some of the latter sources of interoceptive information. In the supine position, for example (Experiment 3), the homeostatic pressure was exerted in a reversed Zaxis direction when pitching backward and forward. When pitching backward, the homeostatic pressure increased significantly at the head level, allowing a fast detection of a change in body orientation. When pitching forward, the variation of hydrostatic pressure at the level of the feet was something natural and less significant for the subjects, as the reference position for the human being is the bipedal stance. In that position (Experiment 2); the varia tion of homeostatic pressure probably did not contribute to the detection of the body tilt because these variations are similar when pitching forward and backward. Other interoceptive signals may have been differently processed by the central nervous system such as the mass of the viscera (von Gierke \& Parker, 1994;Mittelstaedt, 1992,1997; Mittelstaedt \& Fricke, 1988 ), depending on initial body orientation as well as on tilt direction. Following this hypothesis, movements of the viscera would be greater when pitching forward than when pitching backward (Experiment 2). This would result in a lower threshold for the perception of a body tilt. Further experiments(e.g., with paraplegics)need to be carried out to test this interpretation.

These results also indicated that, under specific constraints such as for a complete static posture, the availability of otolith inputs does not imply a better perception of body orientation. From an electrophysiological point of view, it is known that slight modifications of head orientation lead to modulations of the otolith afferents discharge (Fernandez \& Goldberg, 1976). In the present experiment, however, when subjects were tilted at $0.05 \mathrm{deg}$ s, it may be advanced that the speed of variation of the otolithic signal through time was not sufficiently high to have a significant effect on behaviour. Overall, these findings suggest that, at a percep tual level, the otolith organs cannot be considered as efficient graviceptors in quasi-static conditions (Teasdale et al., 1999). The vestibular inputs may become much more useful only when the signal to noise ratio reaches an unambiguous level, such as for more dynamical situa tions (e.g., for more rapid tilts), or with trained subjects, such as gymnasts (Bringoux et al., 2000).

From another point of view, these results also support those of various authors (Fouque et al., 1999; Riccio et al., 1992; Teasdale et al., 1999) showing that the disturbance of dynamic information altered perceived orientation. The ecological theory of orientation suggested that a correct perception of the self-orientation in space requires a dynamic processing of all the available sensory information (Stoffregen \& Riccio, 1988). In the body cast condition, subjects were completely restrained so that they had no active control of any part of their body (except the eyes).By contrast, the level of restraint was reduced in the strapped condition, in which active posturabontrol was not eliminatedlt is then likely that the difference in 
"controllability" between the two conditions of body restriction could account for the differ ence observed in the perceptual responses. According to Stoffregen and Riccio (1988), a higher level of dynamic control of posture generates information that is not available to passive observers. Within the central nervous system, the different sensory inputs have to interact with each other and to be linked to subject's activity, in order to have a significant effect on behaviour. Recent neurophysiological studies support this claim, showing that the activation of vestibular nucleus neurons in response to tilt is not exclusively the result of labyrinthine inputs, but also of limb and trunk afferences (Yates, Jian, Cotter, \& Cass, 2000) as well as efferent signals for postural control (Matsuyama \& Drew, 2000).

In conclusion, the most striking result of the present experiments was that, for very slow velocities, the perception of body orientation was dependent on the characteristics of the avail able sensory inputs with respect to body restriction and initial body position. Contrary to the common assumption, signals issued from the vestibular system were not naturally efficient in quasi-static conditions for accurately perceiving the body orientation with respect to the gravity field. On the other hand, the presentresults confirmed the prominentrole of somesthetic information in postural orientation, such as tactile, proprioceptive (Higashiyama \& Koga, 1998), and interoceptive (Mittelstaedt, 1992) cues.

\section{REFERENCES}

Anastasopoulos, D., Haslwanter, T., Bronstein, A. M., Fetter, M., \& Dichans, J. (1997). Dissociation between the perception of body verticality and the visual vertical in acute peripheral vestibular disorder in humans. Neuroscience Letters, 233, 151-153.

Benson, A. J. (1990). Sensory functions and limitations of the vestibular system. In R. Warren \& A. H. Wertheim (Eds. ), Perception control of self-motion (pp. 145-170). Hillsdale, NJ: Lawrence Erlbaum Associates, Inc.

Bisdorff, A. R., Wolsley, C. J., Anastasopoulos, D., Bronstein, A. M., \& Gresty, M. A. (1996). The perception of body verticality (subjective postural vertical) in peripheral and central vestibular disorders. Brain, 119, 1523-1534.

Bringoux, L., Marin, L., Nougier, V., Barraud P. A., \& Raphel, C. (2000). Effects of gymnastics expertise on the perception of body orientation in the pitch dimension. Journal of Vestibular Research, 10, 251-258.

Bringoux, L., Schmerber, S., Nougier, V., Dumas, G., Barraud, P. A., \& Raphel, C. (2002). Perception of slow pitch and roll body tilts in bilateral labyrinthine-defective subjects. Neuropsychologia, 40, 367-372.

Bronstein, A. M. (1999). The interaction of otolith and proprioceptive information in the perception of verticality. The effects of labyrinthine and CNS disease. Annals of the New York Academy of Sciences, 871, 324-333.

Brown, J. L. (1961). Orientation to the vertical during water immersion. Aerospace Medicine, 32, 209-217.

Clark, B., \& Graybiel, A. (1963). Perception of the postural vertical in normals and subjects with labyrinthine defects. Journal of Experimental Psychology, 65, 490-494.

Dietz, V., Gollhofer, A., Kleiber, M., \& Trippel, M. (1992). Regulation of bipedal stance: Dependence on "load" receptors. Experimental Brain Research, 89, 229-231.

Fernandez, C., \& Goldberg, J. M. (1976). Physiology of peripheral neurons innervating otolith organs of the squirrel monkey. I. Response to static tilts and to long-duration centrifugal force. Journal of Neurophysiology, 39, 970-984.

Fitzpatrick, R., \& McCloskey, D. I. (1994). Proprioceptive, visual, and vestibular threshold for the perception of sway during standing in humans. Journal of Physiology, 1, 173-186.

Fouque, F., Bardy, B. G., Stoffregen, T. A., \& Bootsma, R. J. (1999). Action and intermodal information influence the perception of orientation. Ecological Psychology, 11, 1-43.

Gibson, J. J. (1966). The senses considered as perceptual systems. Boston, MA: Houghton Mifflin.

Goldberg, J. M., \& Fernandez, C. (1984). The vestibular system. In I. Smith (Ed.), Handbook of physiology: Vol. 3. The nervous system (pp. 977-1021). New York: Academic Press.

Higashiyama, A., \& Koga, K. (1998). Apparent body tilt and postural aftereffect. Perception \& Psychophysics, 60, 331347.

Howard, I. P. (1997). Interactions within and between the spatial senses. Journal of Vestibular Research, 7, 311-345. 
Lackner, J. R., \& DiZio, P. (1993). Mutisensory, cognitive, and motor influences on human spatial orientation in weightlessness. Journal of Vestibular Research, 3, 361-372.

Lackner, J. R., \& DiZio, P. (2000). Human orientation and movement control in weightless and artificial gravity environments. Experimental Brain Research, 130, 2-26.

Lackner, J. R., \& Graybiel, A. (1978). Some influences of touch and pressure cues on human spatial orientation. Aviation, Space and Environmental Medicine, 49, 798-804.

Lestienne, F., \& Gurfinkel, V. S. (1988). Postural control in weightlessness: A dual process underlying adaptation to an unusual environment. Trends in Neurosciences, 11, 359-363.

Massion, J., Fabre, J. C., Mouchnino, L., \& Obadia, A. (1995). Body orientation and regulation of the center of gravity during movement under water. Journal of Vestibular Research, 5, 211-221.

Matsuyama, K., \& Drew, T. (2000). Vestibulospinal and reticulospinal neuronal activity during locomotion in the intact cat. I. Walking on a level surface. Journal of Neurophysiology, 84, 2237-2256.

Merfeld, D. M., Young, L. R., Oman, C. M., \& Shelhamer, M. J. (1993). A multidimensional model of the effect of gravity on the spatial orientation of the monkey. Journal of Vestibular Research, 3, 141-161.

Merfeld, D. M., Zupan, L., \& Peterka, R. J. (1999). Humans use internal models to estimate gravity and linear acceleration. Nature, 398, 615-618.

Mergner, T., \& Glasauer, S. (1999). A simple model of vestibular canal-otolith signal fusion. Annals of the New York Academy of Sciences, 871, 430-434.

Mittelstaedt, H. (1992). Somatic versus vestibular gravity reception in man. Annals of the New York Academy of Sciences, 656, 124-139.

Mittelstaedt, H. (1997). Interaction of eye-, head-, and trunk-bound information in spatial perception and control. Journal of Vestibular Research, 7, 283-302.

Mittelstaedt, H. (1999). The role of otoliths in perception of the vertical and in path integration. Annals of the New York Academy of Sciences, 871, 334-343.

Mittelstaedt,H., \& Fricke, E. (1988).The relative effect of saccular and somatosensory information on spatial orientation and control. Advances in Oto-Laryngology, 42, 24-30.

Nelson, J. G. (1968). Effect of water immersion and body position upon position of gravitational vertical. Aerospace Medicine, 39, 806-811.

Nemire, K., \& Cohen, M. M. (1993). Visual and somesthetic influences on postural orientation in the median plane. Perception \& Psychophysics, 53, 106-116.

Riccio, G. E., Martin, E. J., \& Stoffregen, T. A. (1992). The role of balance dynamics in the active perception of orientation. Journal of Experimental Psychology: Human Perception and Performance, 3, 624-644.

Ross, H. E. (1990). Orientation and movement in divers. In R. Warren \& A. H. Wertheim (Eds.), Perception control of self-motion (pp. 463-486). Hillsdale, NJ: Lawrence Erlbaum Associates, Inc.

Ross, H. E., Crickmar, S. D., Sills, N. V., \& Owen, E. P. (1969). Orientation to the vertical in free divers. Aerospace Medicine, 40, 728-732.

Stoffregen, T. A., \& Bardy, B. G. (2001). On specification and the senses. Behavioral and Brain Sciences, 24, $195-261$.

Stoffregen, T. A., \& Riccio, G. E. (1988). An ecological theory of orientation and the vestibular system. Psychological Review, 95, 3-14.

Teasdale, N., Nougier, V., Barraud, P. A., Bourdin, C., Debû, B., Poquin, D., \& Raphel, C. (1999). Contribution of ankle, knee and hip joints to the perception threshold for support surface rotation. Perception \& Psychophysics, 61, 615-624.

von Gierke, H. E., \& Parker, D. E. (1994). Differences in otolith and abdominal visceria graviceptor dynamics: Implications for motion sickness and perceived body position. Aviation, Space and Environmental Medicine, 65, 747-751.

Yates, B. J., Jian, B. J., Cotter, L. A., \& Cass, S. P. (2000). Responses of vestibular nucleus neurons to tilt following chronic bilateral removal of vestibular inputs. Experimental Brain Research, 130, 151-158.

Young, L. R. (1984). Perception of the body in space: Mechanisms. In I. Smith (Ed.), Handbook of physiology: Vol. 3. The nervous system (pp. 1023-1066). New York: Academic Press. 